\title{
Valoración de la función renal en diabéticos tipo 2 y su adecuación al tratamiento antidiabético oral
}

\author{
José Juan Carbayo García ${ }^{a}$, Roxana Tuesta Reinab ${ }^{b}$ José Félix Sastre Garcíac, Juan José \\ Criado Álvarez ${ }^{\mathrm{d}}$, Carlos Gómez Gonzáleze ${ }^{\mathrm{e}}$ Jesús Rodríguez Losáñez ${ }^{\mathrm{e}}$.
}

\author{
a Médico de Familia, C.S. \\ Talavera Centro. Gerencia de \\ Atención Integrada de Talavera \\ de la Reina (Toledo) \\ ${ }^{\mathrm{b}}$ Técnico de Salud. Unidad \\ Docente de Medicina de \\ Familia y Comunitaria de \\ Talavera de la Reina (Toledo). \\ ${ }^{\mathrm{c}}$ Médico de Familia, C.S. \\ Los Navalmorales. Gerencia de \\ Atención Integrada de Talavera \\ de la Reina (Toledo). \\ ${ }^{d}$ Médico de Familia, C.S. \\ La Pueblanueva. Gerencia de \\ Atención Integrada de Talavera \\ de la Reina (Toledo). \\ e Médico Residente de \\ Medicina Familiar y \\ Comunitaria. Centro de Salud \\ Talavera Centro. \\ Correspondencia: \\ José Juan Carbayo García. \\ Centro de Salud Talavera \\ Centro. \\ C/ José Luis Gallo n 2. \\ 45600. Talavera de la Reina. \\ Toledo. \\ Correo electrónico: \\ jjcarbayo@sescam.jccm.es
}

Recibido el 27 de julio de 2013. Aceptado para su publicación el 3 de febrero de 2014.

\section{RESUMEN}

Objetivo: Determinar la función renal mediante filtrado glomerular (FG) en diabéticos tipo 2 y su adecuación al tratamiento antidiabético oral.

Material y Método: Estudio observacional transversal realizado en una muestra de diabéticos tipo 2, con una edad de 18 a 75 años, en tratamiento con antidiabéticos orales (ADO), en el Área Sanitaria de Talavera de la Reina (Toledo), en el periodo noviembre 2011 a junio de 2012.

Resultados: Se estudiaron 191 pacientes, 103 varones, 88 mujeres, con edad media $62,5(8,3)$ años. A $168(87,96 \%)$ de estos pacientes se les pudo calcular el FG, siendo el FG medio de 95,5 $(25,1)$. Siete $(3,7 \%)$ pacientes en rango $45-60$ y uno $(0,5 \%)$ con FG menor de 30 . La prevalencia de enfermedad renal crónica (ERC) fue del 4,8\% [IC95\% 2,4-9,1].

El fármaco más utilizado fue metformina seguido de IDPP4. En relación al FG en rango 4560 , los más utilizados fueron los inhibidores DPP4 solos o combinados con metformina; en el paciente con FG menor de 30, el medicamento utilizado fue la metformina.

Conclusiones: La prevalencia que hemos hallado es inferior a la de otros estudios publicados. Es importante la determinación del FG en pacientes diabéticos. En nuestros pacientes, las indicaciones de tratamiento se adecuan, en general, a la función renal.

Palabras clave: Enfermedad renal crónica. Diabetes Mellitus. Filtrado glomerular. Antidiabéticos orales. Atención Primaria de Salud.

\section{ABSTRACT}

Assessment of the kidney function in type 2 diabetic patients and their appropriateness for oral antidiabetic treatment.

Objective: Assessment of the kidney function by glomerular filtrate rate (GFR) in type 2 diabetic subjects and their appropriateness for antidiabetic oral treatment.

Materials and Method: Cross-sectional observational study carried out in a sample of type 2 diabetic subjects aged between 18 and 75 years old treated with oral antidiabetic drugs (OADs) in the Health area of Talavera de la Reina (Toledo, Spain) in the period from November 2011 to June 2012.

Results: A group of 191 pacients was studied: 103 men and 88 women, with an average age of 62.5 (8.3) years. The FGR was calculated in 168 patients (87.96\%), obtaining an FGR average value of 95.5 (25.1). Seven patients $(3.7 \%)$ were within the $45-60$ range and one $(0.5 \%)$ with FGR under 30 . The prevalence of chronic kidney disease (CKD) was 4.8\% [CI95\% 2, 4-9,1].

The most widely used drug was metformine followed by IDPP4. In relation to GFR in the 45-60 range, the most frequent drugs were DPP4 inhibitors alone or combined with metformine; in the patient with FGR below 30 , the drug used was metformine.

Conclusions: The prevalence found was lower than that in other previous studies published. The estimation of FGR is important in diabetic patients. In our group of patients, the treatment guidelines are generally adjusted to the kidney function.

Keywords: Chronic kidney disease, Diabetes mellitus. Glomerular filtration. Oral antidiabetic drugs. Primary health care. 


\section{INTRODUCCIÓN}

La diabetes mellitus (DM), por su elevada prevalencia $(4,8-18,7 \%)$ y su enorme trascendencia vital en la salud y en la calidad de vida, es considerada actualmente uno de los mayores problemas de salud pública ${ }^{1}$.

Las diversas estrategias de intervención se han mostrado altamente eficaces, con una relación coste/beneficio muy favorable, el coste de las complicaciones es tres veces superior al tratamiento y control de las alteraciones metabólicas ${ }^{1,2}$.

Disponemos de un amplio arsenal farmacológico avalado por evidencia científica, consensos, guías clínicas y sociedades científicas ${ }^{3-5}$. La elección de uno $\mathrm{u}$ otro fármaco debe basarse en las características del paciente, la existencia de patologías asociadas y las contraindicaciones de dicho medicamento.

La enfermedad renal crónica (ERC) se define como la disminución de la función renal, expresada por un filtrado glomerular (FG) menor de $60 \mathrm{ml} /$ $\mathrm{min} / 1,73 \mathrm{~m}^{2}$ o como la presencia de daño renal de forma persistente durante al menos 3 meses $^{6}$.

Un número importante de pacientes con ERC están sin diagnosticar, bien porque no se efectúan controles de función renal, o bien porque tienen una ERC oculta (tienen enfermedad renal a pesar de que las creatininas séricas están en el rango de normalidad del laboratorio) ${ }^{7}$. En pacientes seguidos en atención primaria con enfermedades tan frecuentes como la HTA o la diabetes mellitus, la prevalencia de insuficiencia renal puede alcanzar cifras del 5,4 al $21,4 \%{ }^{8}$. La determinación de creatinina sérica no debe ser utilizada como único parámetro para evaluar la función renal. La estimación del FG a través de ecuaciones es el mejor índice disponible en la práctica clínica para evaluar la función renal ${ }^{9}$. En la actualidad, el MDRD-4, debido a su facilidad de implementación en los informes de laboratorio y sensibilidad en la detección precoz de la ERC, es la ecuación recomendada por la mayoría de sociedades científicas ${ }^{9}$.

Dado que la prevalencia de nefropatía diabética es elevada ${ }^{8}$, que es indispensable la determinación del FG para conocer la función renal y para establecer su estadío evolutivo, y que en el abordaje global del paciente con ERC debe ponerse, además, especial atención en evitar la iatrogenia ajustando los fármacos al FG, en este trabajo queremos conocer la función renal mediante la determinación del filtrado glomerular en los diabéticos tipo 2 de nuestra área de salud y en qué grado el tratamiento antidiabéti- co oral se adapta a las recomendaciones establecidas.

\section{MATERIAL Y MÉTODOS}

Se trata de un estudio observacional transversal, realizado en una población de pacientes diabéticos tipo 2 atendidos en Atención Primaria del área de Salud de Talavera, en tratamiento con antidiabéticos orales (ADO). Como criterios de inclusión se utilizaron los siguientes: diabéticos tipo 2 en tratamiento con $A D O$, edad entre 18 y 75 años y tener realizada analítica en el año 2011 con los parámetros necesarios para calcular el FG. El estudio fue realizado entre noviembre de 2011 y junio de 2012.

El tamaño muestral se calculó para una población de 65.000 habitantes entre 18 y 75 años, considerando una prevalencia de diabetes tipo 2 del 12\% y una precisión de $\pm 5 \%$, y un nivel de confianza del $95 \%$, resultando 162 pacientes, sobre la cual se calculó un porcentaje de pérdidas del $15 \%$, siendo el tamaño final de la muestra de 187 pacientes.

Se obtuvo un listado del total de pacientes con los diagnósticos de diabetes tipo 2 no descompensada y diabetes tipo 2 descompensada (códigos CIE9250.90 y 250.92) de la base de datos Turriano del SESCAM. El muestreo se ha realizado utilizando la generación de números aleatorios del programa Excel 2003 aplicada al total de pacientes entre 18 y 75 años con diagnóstico de diabetes tipo 2 en tratamiento con antidiabéticos orales durante el periodo noviembre 2011 - junio 2012.

La variables recogidas en cada paciente fueron: edad, sexo, talla, peso, valor de creatinina, fármacos utilizados, índice de masa corporal (considerando peso / talla $[\mathrm{m}]^{2}$ ) y la tasa de filtrado glomerular, utilizando para su cálculo la ecuación MDRD4 con la calculadora facilitada por la Sociedad Española de Nefrología, que incluye las variables edad, peso, talla, sexo, creatinina, urea, albúmina y etnia afroamericana.

En el análisis estadístico, se ha calculado las medidas de tendencia central y de dispersión: media, desviación estándar e intervalos de confianza de las variables cuantitativas más importantes, las frecuencias de las variables cualitativas. Se ha utilizado el test de Kruskal Wallis para comparar las medias de creatinina, filtrado glomerular e IMC según sexo y grupo de edad, y se ha calculado la frecuencia del fármaco prescrito según la tasa de filtrado glomerular. Se han utilizado los programas Excel 2003 y SPSS 17.0 y se ha considerado un nivel de significación del 0.05 . 


\section{RESULTADOS}

Se estudiaron 191 pacientes diagnosticados de DM2 tratados con ADO, 103 eran varones $(53,9 \%)$ y 88 mujeres $(46,1 \%)$. La edad media fue de 62,5 (DE: 8,3) años. A $168(87,9 \%)$ de estos pacientes se les pudo calcular el FG, siendo el FG medio de 95,5 (DE: 25,1$) \mathrm{mL} / \mathrm{min} / 1,73 \mathrm{~m}^{2}$ (tabla 1).

La distribución de la tasa de filtrado glomerular se acerca a la normal con una ligera desviación a la derecha (figura 1). Siete pacientes $(3,7 \%)$ tuvieron un $F G$ en rango 45-60, no hubo ninguno con $F G$ en el rango 30-44 y uno (0,5\%) presentó un FG menor de 30 .

La prevalencia de ERC fue del $4,8 \%$, con un intervalo de confianza del $95 \%$ de $2,4-9,1 \%$.

El principio activo más prescrito ha sido metformina. En 41 pacientes no consta el tratamiento (tabla 2). Según tipo de antidiabético y tasa de filtrado glomerular, el ADO más utilizado en todos los grupos fue la metformina sola o combinada (98 pacientes,
$72 \%)$, seguido de los inhibidores de la dipeptidil peptidasa 4 o IDPP4 (27 pacientes, 19,8\%); en 10 pacientes $(7,3 \%)$ el ADO prescrito se asociaba a insulina. En 14 pacientes no constaban los valores de creatinina por lo que no se calcularon las tasas de filtrado glomerular (tabla 3).

La diferencia de medias del índice de masa corporal y la tasa de filtrado glomerular según grupo de edad fueron significativas tanto en hombres como en mujeres; mientras que la diferencia de medias de creatinina según grupo de edad fue significativa sólo en mujeres (tabla 4).

\section{DISCUSIÓN}

Gracias a la clasificación en estadios de la enfermedad renal crónica propuesta por la National Kidney Foundation ${ }^{6}$ en el año 2002, que permitió unificar criterios y comparar estudios poblacionales, se sabe que la ERC es muy prevalente. Según los datos del estudio EPIRCE ${ }^{8}$, la prevalencia de

\begin{tabular}{lccccc}
\hline Variable & Media (DE) & & Mínimo & & Máximo \\
\cline { 5 - 6 } \cline { 5 - 6 } Edad (años) & $62,45(8,31)$ & & 38,23 & & 75,94 \\
Índice de masa corporal & $31,5(6,22)$ & & 20,18 & & 56,64 \\
Creatinina en sangre (mg/dl) & $0,83(0,26)$ & & 0,41 & & 3,20 \\
Función renal (MDRD4) & $95,46(25,1)$ & & 20,40 & & 175,63 \\
\hline
\end{tabular}

Tabla 1. Medidas de tendencia central y de dispersión

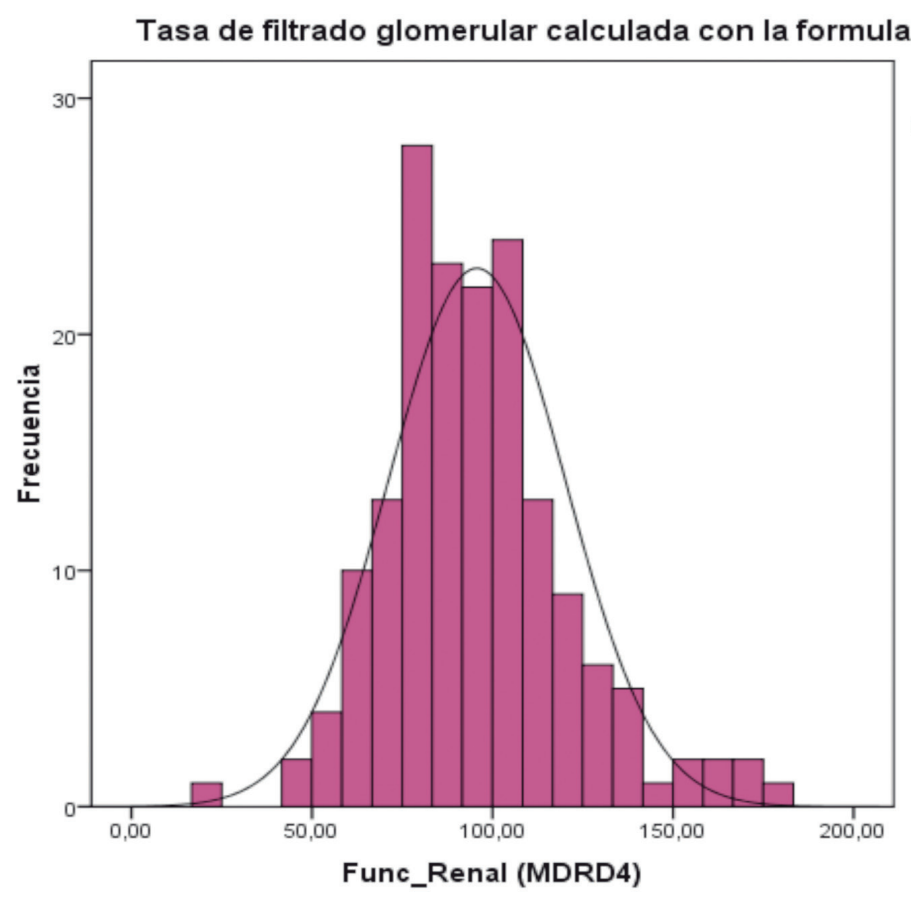

Figura 1. Histograma de la tasa de filtrado glomerular calculada utilizando la ecuación MDRD-4 
ERC en la población española fue del $6,8 \%$, con un intervalo de confianza del $95 \%$ de 5,4 a 8,2 (3,3\% para edades $40-64$ años y $21,4 \%$ para edades $>64$ años) y de elevada morbilidad vascular.

El conocimiento de su prevalencia en la población general puede contribuir a la detección precoz de esta enfermedad y a prevenir o retrasar su evolución. Además, ha permitido confirmar la relación estrecha entre enfermedad cardiovascular y ERC, mayor que la simplemente casual por compartir factores de riesgo y de progresión comunes. De hecho, las guías clínicas y los documentos de consenso recientes, así como las guías sobre el manejo y el tratamiento del paciente con hipertensión arterial, reconocen como importantes factores de riesgo cardiovascular tanto al descenso del filtrado glomerular (FG) como a la albuminuria².

En nuestro estudio, la prevalencia global fue del $4,8 \%$, para pacientes diabéticos con edades comprendidas entre los 18 y 75 años, edades para las que está validada la fórmula MDRD. Se trata de un porcentaje inferior al resto de la población española y de otros países del mismo entorno geográfico. Las razones para explicarlo pueden ser el mero azar, la edad de inclusión que hemos considerado, que excluye a los mayores de 75 años, en los que la enfermedad renal es más prevalente, o el sesgo de inclusión de los pacientes, ya que existen múltiples diagnósticos de diabetes en el sistema informático utilizado por nuestro Servicio de Salud y, aunque se han utilizado los más generales, pueden haberse excluido algunos pacientes con enfermedad renal.
Es importante la determinación del FG, es el criterio adoptado para la valoración de la función renal y evita las ERC ocultas que ocurren con la sola utilización de creatinina. En la práctica clínica, esta valoración se hace mediante fórmulas basadas en la creatinina sérica. La fórmula más utilizada hasta la fecha es la MDRD-4 o la MDRD-IDMS ${ }^{9,10}$. Sin embargo, hay que tener siempre presente que todas estas fórmulas son estimaciones y que siempre deben interpretarse en el contexto clínico de cada paciente. Nosotros hemos utilizado la misma que nuestro laboratorio de referencia MDRD-4.

Además, para el diagnóstico de ERC es imprescindible no sólo estimar el FG, sino medir la albuminuria, ya que ésta, aparte de ser un importante factor de riesgo vascular, es el principal marcador de progresión de la propia enfermedad renal ${ }^{9}$.

En la actualidad disponemos de un amplio arsenal terapéutico para el tratamiento de los pacientes con diabetes mellitus tipo 2 (DM2), que incluye metformina, sulfonilureas, glinidas, tiazolidindionas, inhibidores de las disacaridasas, inhibidores de la dipeptidil-peptidasa (IDPP-4) y antagonistas del receptor del péptido 1 similar al glucagón (GLP1), que junto con la insulina pueden utilizarse en monoterapia o en asociación. Estos fármacos deben utilizarse tras la consideración de sus respectivas fichas técnicas.

La elección del tratamiento depende de múltiples aspectos relacionados con las características del paciente, la potencia del fármaco para lograr el objetivo terapéutico, las complicaciones o enferme-

\begin{tabular}{lrr}
\hline Principio Activo & \multicolumn{1}{c}{$\mathbf{n}^{*}$} & \% \\
\cline { 3 - 3 } \cline { 3 - 3 } Metformina & 83 & 55,33 \\
Sitagliptina - Metformina & 18 & 12,00 \\
Repaglinida & 16 & 10,67 \\
INSULINA & 11 & 7,33 \\
Glicazida & 6 & 4,00 \\
Vidagliptina - Metformina & 6 & 4,00 \\
Glibenclamida & 4 & 2,67 \\
Sitagliptina & 3 & 2,00 \\
Glizipida & 1 & 0,67 \\
Pioglitazona & 1 & 0,67 \\
Saxagliptina & 1 & 0,67 \\
Total & 150 & 100 \\
\hline
\end{tabular}

Tabla 2. Frecuencia absoluta y relativa según principio activo *No consta tratamiento en 41 de los 191 casos estudiados. 


\begin{tabular}{lcccc}
\hline \multicolumn{1}{c}{ Fármaco grupo } & \multicolumn{2}{c}{ Tasa de filtrado glomerular $\left(\mathbf{m l} / \mathbf{m i n} / \mathbf{1 , 7 3} \mathbf{~ m}^{2}\right)$} & Total \\
\hline Metformina & $\mathbf{6 0}$ & $\mathbf{4 5 - 6 0}$ & $<\mathbf{3 0}$ & 75 \\
Inhibidores DPP4 + & 73 & 1 & 1 & 23 \\
Metformina & 22 & 1 & - & 14 \\
Glinidas & 14 & - & - & 10 \\
Insulina & 9 & 1 & - & 9 \\
Sulfonilureas & 9 & - & - & 4 \\
Inhibidores DPP4 & 2 & 2 & - & 1 \\
Tiazolindionas & 1 & - & - & 136 \\
Total general & 130 & 5 & 1 & \\
\hline
\end{tabular}

Tabla 3. Fármacos utilizados según tasa de filtrado glomerular

dades asociadas, el riesgo de efectos adversos, la tolerancia y el coste $e^{1,11}$.

Las principales guías y documentos de consenso nacionales e internacionales para el tratamiento de la $\mathrm{DM} 2^{3-5}$ recomiendan la utilización de metformina como una primera opción farmacológica, sin que exista un criterio uniforme sobre su empleo en pacientes con distintos estadios de insuficiencia renal. Sin embargo, no existe la misma unanimidad en considerar a partir de qué nivel de daño renal su uso estaría contraindicado en base al potencial riesgo de desarrollar acidosis láctica, una rara pero grave complicación que puede acompañarla ${ }^{12,13}$.

En general, se establece el empleo de metformina en pacientes con una tasa de filtrado glomerular (TFG) entre 45 y $60 \mathrm{ml} / \mathrm{min}$, realizando controles semestrales de función renal, y en pacientes con TFG entre 30 y $45 \mathrm{ml} / \mathrm{min}$, reduciendo la dosis a la mitad y controlando la función renal cada 3 meses, manteniéndose la contraindicación absoluta para filtrados inferiores a $30 / \mathrm{ml} / \mathrm{min} .{ }^{14}$ Es reseñable que uno de los pacientes de nuestro estudio con $\mathrm{FG}<30$ estaba en tratamiento con metformina.

La eliminación de repaglinida es principalmente biliar, por lo que está aceptado su uso en cualquier estadio de ERC, incluso en pacientes en diálisis ${ }^{12}$. Llama la atención no haber sido utilizada en FG < 60 en nuestro estudio.

Los IDPP4 comercializados en España en el momento actual son sitagliptina, vildagliptina, saxa- gliptina y linagliptina. Este grupo farmacológico presenta la gran ventaja frente a los secretagogos clásicos de producir menos hipoglucemias, dado que su mecanismo estimulador de la secreción de insulina es dependiente de la glucosa ${ }^{12}$, característica que los hace especialmente atractivos en presencia de ERC por la predisposición de estos pacientes a desarrollar hipoglucemias ${ }^{15}$. Sitagliptina, vildagliptina y saxagliptina precisan ajuste de dosis, dado que su eliminación es fundamentalmente renal $^{12}$. En el estudio se utilizaron en tres pacientes con $\mathrm{FG}<60$, a dosis estándar, sin que conste la realización de dicho ajuste.

Los estudios recientes con linagliptina muestran que su eliminación es hepatobiliar y su vida media apenas se prolonga, en la ERC avanzada, un máximo de 14 a 18 horas, por lo que no precisa ajuste de dosis, habiendo sido incluso utilizada en pacientes diabéticos en hemodiálisis ${ }^{12}$.

Como conclusiones del estudio podríamos decir que, aunque la prevalencia hallada es inferior a la de otros estudios publicados, podría deberse al tamaño muestral limitado o a los posibles sesgos arriba comentados, y que en nuestros pacientes las indicaciones de tratamiento se adecuan en general a la función renal, siendo los fármacos más utilizados la metformina, los IDPP4 y la repaglinida.

Por su importancia, creemos que el registro del FG en las historias clínicas debería tener un lugar claramente establecido; en nuestro trabajo ha sido necesario calcularlo a partir de las variables precisas y los datos de laboratorio. 


\begin{tabular}{lcccc}
\hline \multicolumn{1}{c}{ Grupo edad (años) } & $\mathbf{3 5 - 4 5}$ & $\mathbf{4 6 - 6 5}$ & $\mathbf{> 6 5}$ & $\boldsymbol{p}$ \\
\cline { 2 - 5 } & \multicolumn{4}{c}{ Hombres } \\
\cline { 2 - 5 } Índice de masa corporal (media y DE) & $35,77(5,57)$ & $31,29(4,81)$ & $28,87(4,69)$ & 0,009 \\
Creatinina (media y DE) & $0,73(-)$ & $0,87(0,16)$ & $0,97(0,41)$ & 0,347 \\
Filtrado glomerular (media y DE) & $138,49(16,98)$ & $100,57(25,01)$ & $90,15(26,12)$ & 0,030 \\
\hline & \multicolumn{4}{c}{ Mujeres } \\
Índice de masa corporal (media y DE) & $41,87(13,11)$ & $33,76(7,22)$ & $30,95(5,88)$ & 0,061 \\
Creatinina (media y DE) & $0,7(0,07)$ & $0,68(0,16)$ & $0,77(0,16)$ & 0,017 \\
Filtrado glomerular (media y DE) & $98,96(10,88)$ & $102,18(25,5)$ & $83,07(17,66)$ & 0,001 \\
\hline
\end{tabular}

Tabla 4. Índice de masa corporal, creatinina y filtrado glomerular según sexo y edad

\section{BIBLIOGRAFÍA}

1. Ruiz - Ramos M, Escolar - Pujolar A, Mayoral - Sánchez E, Corral - San Laureano F, Fernández - Fernández I, La diabetes mellitus en España: mortalidad, prevalencia, incidencia, costes económicos y desigualdades. Gac Sanit. 2006; 20 (Supp1):15-24.

2. National Health and Medical Research Council. National evidence based guidelines for blood glucose control in type 2 diabetes. Disponible en: http://www.nhmrc.gov.au/ guidelines/publications/di19 [consultado el 10/11/2011]

3. Menéndez-Torre E, Lafita Tejedor J, Artola Menéndez S, Millán Núñez-Cortes J, Alonso García A, Puig Domingo M, et al. Recomendaciones para el tratamiento farmacológico de la hiperglucemia en la diabetes mellitus tipo 2. Av Diabetol. 2010; 26:331-8.

4. Inzucchi SE, Bergenstal RM, Buse JB, Diamant M, Ferrannini $E$, Nauck $M$, et al. Management of hyperglycemia in type 2 diabetes: a patient-centered approach: position statement of the American Diabetes Association (ADA) and the European Association for the Study of Diabetes (EASD). Diabetes Care. 2012;35(6):1364-79.

5. National Institute of Health and clinical Excellence. Type 2 diabetes: the management of type 2 diabetes: NICE clinical guideline 87. 2009. Disponible en: http://guidance.nice.org. uk/CG87/Guidance [consultado el 10/11/2011]

6. National Kidney Foundation. K/DOQI clinical practice guidelines for chronic kidney disease: evaluation, classification, and stratification. Am J Kidney Dis. 2002;39(2 Suppl 1):S1-266.

7. Alcázar R, Egocheaga MI, Orte L, Lobos JM, González Parra E, Álvarez Guisasola F, Górriz JL, Navarro JF, Martín de Francisco AL. Documento de consenso SEN-SEMFYC sobre la enfermedad renal crónica. Nefrología. 2008; 28(3)273-282.
8. Otero A, De Francisco ALM, García F. Prevalencia de la insuficiencia renal crónica en España: Resultados del estudio EPIRCE. Nefrología. 2010;30:78-86.

9. Gracia S, Montañés R, Bover J, Cases A, Deulofeu R, Martín de Francisco AL, Orte LM; Sociedad Española de Nefrología. Documento de consenso: Recomendaciones sobre la utilización de ecuaciones para la estimación del filtrado glomerular en adultos. Nefrología. 2006; 26(6) 658- 665.

10. Levey AS, Stevens LA, Schmid CH, Zhang YL, Castro AF, Feldman $\mathrm{HI}$, et al. A new equation to estimate filtration rate. Ann Intern Med. 2009;150:604-12.

11. Pozo-Fernández $C$, Pardo-Ruiz $C$, Sánchez-Botella $C$, Blanes-Castañer V, López-Menchero R, Gisbert-Sellés C, et al. Discrepancias entre documentos de consenso, guías, práctica clínica y normativa legal en el tratamiento de los pacientes con diabetes mellitus tipo 2. Nefrología. 2012;32:367-73.

12. Agencia Española de Medicamentos y Productos Sanitarios. Ficha Técnica de medicamentos. Disponible en: $\quad$ http://www.aemps.gob.es/cima/fichasTecnicas. do?metodo=detalleForm [consultado el 10/11/2011]

13. Nathan DM, Buse JB, Davidson MB, Ferrannini E, Holman RR, Sherwin R, et al. Medical Management of hyperglycemia in type 2 diabetes: a consensus algorithm for the initiation and adjustment of therapy. Diabetes Care. 2009;32:193-203.

14. Martínez-Castelao A, Górriz JL, Sola E, Morillas C, Jover A, Coronel F, et al. A propósito de las discrepancias entre documentos de consenso, guías de práctica clínica y normativa legal en el tratamiento de la diabetes tipo 2. Nefrología. 2012;32(4):419-26.

15. Górriz JL, De Alvaro F, Martínez-Castelao A. Tratamiento del paciente diabético con Insuficiencia Renal. Nefrología. 2001;21(3):66-75. 